\title{
Seed-Germination and Seedling-Growth Inhibition by Aqueous Extracts from Hyptis Rhomboidea
}

\author{
Ling Wang ${ }^{1}$, De-ying Deng ${ }^{1}$, Lu Tang ${ }^{1}, \mathrm{Ke} \mathrm{Yuan}^{2}, \mathrm{Yu}$-xian $\mathrm{Li}^{1}$ \\ ${ }^{1}$ College of Pharmacy, Henan University of Traditional Chinese Medicine, Zhengzhou 450008, P.R. China; \\ ${ }^{2}$ Zhejiang Agriculture and Forestry University, Lin' an, China \\ *Corresponding author
}

ABSTRACT: To evaluate the allelopathic potential of Hyptis rhomboide Mart. et Gal (native to tropical America ), the effects of aqueous extracts $(0.0125,0.025,0.05,0.1 \mathrm{~g} / \mathrm{mL})$ prepared from stalks and leaves of H. rhomboide were studied on the seed germination and seedling growth of four crops (Brassica campestris, Raphanus sativus L., Oryza sativa, and Lactuca sativa).The results showed that the two aqueous extracts significantly inhibited the seed germination and seedling growth of Brassica campestris, Raphanus sativus L., Oryza sativa at the higher concentrations $(0.05,0.1 \mathrm{~g} / \mathrm{mL})$, and emerged positive relative with concentration, whereas the lower concentrations had a positive effect on the seed germination and seedling growth of Lactuca sativa by the leaf extracts. By synthetical effect, both the two extracts caused the four receiver seeds with low promote high inhibit effect. The stalks aqueous extracts showed the greatest inhibition effect on the Raphanus sativus L, the leaf showed the greatest inhibition effect on the Brassica campestris and the maximum allelopathic effect occurred with leaf extracts. The results suggested that stalks or leaves extracts of $\mathrm{H}$. rhomboide may influence the natural crops with in allelopathic y effect via the release of allelochemicals from the aerial parts into the environment. The results demonstrate that the aqueous extracts from $\mathrm{H}$. rhomboide allelopathic potential and should be evaluated as an allelopathic species, presenting a risk or advantage to seed germination and seedling growth of crop or weed plants.

KEYWORD: Hyptis rhomboidea; aqueous extracts; seed germination; seedling growth; allelopathic

\section{INTRODUCTION}

The allelopathy of plants, which release some special chemicals to the environment through stalks, leaves and root, acts on the other plants or themselves direct or indirect beneficial or harmful effect[1-3]. Allelopathy exists widely in the nature, and it's a natural chemical regulation phenomenon in order to adapt environment, Studying the allelopathy have the great significance in the formation and succession of vegetation, and plant growth[4-5].

Hyptis rhomboidea Mart. et Gal is also called Pycnanthemum decurrens Blanco, Hyptis decurrens (Blanco) Epling, Hyptis celebica. The Hyptis genus is a member of the Lamiaceae family and includes about 775 species, it is native to subtropical area of American and the West Indies, but now spreads the tropical all over the world. In China, it is almost grown in Guangdong, Guangxi, Hainan, Hongkong, Taiwan as weed. Research on $H$. rhomboidea is rarely reported. this article has done some primary allelopathy about the aerial parts of $H$. rhomboidea. Aimed at revealing allelopathy effect when the plants invasiving, and its effects on seed germination and plants growth, and providing for making full useful wild plants.

\section{EXPERIMENTAL}

The aerial parts of $H$. rhomboidea were collected from Sanya of Hainan province in China in June 2011. And it was identified by Huang Shiman, Medicinal Plants Taxonomy Professor. The four receiver plants were Brassica campestris, Raphanus sativus L., Oryza sativa, and Lactuca sativa respectively. And both were bought in lin'an seeds company.

Follow the literature with modification[6]: In order to imitate natural condition cut off the flowers and seeds. Watered the fresh stalks and leaves, dried them at room temperature, cut into $1 \sim 2 \mathrm{~cm}$ segments. Took the 1:10 with distilled water, quenched $48 \mathrm{~h}$ temperature, slight shaking every six hours, double filtration, the first filtration with qualitative filter paper, and the second with $0.45 \mu \mathrm{m}$ membrane, got 
stalks and leaves' mother liquor. Diluted the two aqueous extracts with distilled water to $0.1,0.05$, $0.025,0.0125 \mathrm{~g} / \mathrm{mL}$ respectively. Storage at $4{ }^{\circ} \mathrm{C}$ for later use.

Petri dish, beaker and filter paper were sterilized at $0.5 \mathrm{~Pa}$ for $30 \mathrm{~min}$, cool for later use. Take seeds into $0.3 \% \mathrm{KMnO}_{4}$ for $15 \mathrm{~min}$. Rinsing with distilled water to colorless, dry them for later use.

Seed germination and seedling growth trial: Selected the full seeds, placed in Petri dish with two pieces of filter paper, 20 grains, $3 \mathrm{~mL}$ different concentration extracts per Petri dish, equal water in control group, three parallel every handles . Supplement certain extracts and water daily. Temperature is $25^{\circ} \mathrm{C}$, the humidity is $70 \%$. Counted the seed germination number every $24 \mathrm{~h}$ until the seventh day.

According to the method of the literature [7] with some modifications: With "little beaker method", the beaker with two pieces of filter paper as a incubator, placed 4 similar growth seedlings per beaker, then added $1 \mathrm{~mL}$ different concentration extracts and distilled water for groups, sealed with parafilm in case of water evaporation and influences with different groups. Placed the beakers with seedlings at constant temperature of $25{ }^{\circ} \mathrm{C}, 12 \mathrm{~h} / \mathrm{d}$ light incubator, three parallel per deals, and water group as the control. Measured seedlings' root, shoot length, and root, shoot weight and the fresh and dry weight $\left(30 \mathrm{~min}\right.$ at $125^{\circ} \mathrm{C}$, then low to $75^{\circ} \mathrm{C}$ to constant weight ).

Germination rate $(\%)=$ (number of seed germination at a set time / the total number of seeds $) \times 100 \%$. Germination index $=\Sigma(\mathrm{Gt} / \mathrm{Dt})[8]$, where the Gt is the number of seed germination on the $\mathrm{t}$-th day, and the Dt is the corresponding germination day. Radicle length and elongation of the aerial portion of seedlings of target species were recorded weekly from the Petri dishes and then we compared measurements derived from seedlings of the same age. Dry weight of seedlings at the end of the experiment was also recorded.

Allelopathic effect index (RI) was put forward to measure the allelopathy intensity index: $\mathrm{RI}=1$ $\mathrm{C} / \mathrm{T}(\mathrm{T} \geq \mathrm{C}), \quad \mathrm{RI}=\mathrm{T} / \mathrm{C}-1(\mathrm{~T}<\mathrm{C})$, where the $\mathrm{C}$ is the control value and the $\mathrm{T}$ is the process value .when $\mathrm{RI}>0$, it means promotion and $\mathrm{RI}<0$, it means inhibition .The magnitude of the RI's absolute value stands for the allelopathy strength of the extracts effect on seeds. Synthetic Effect[9] is a arithmetic mean of several test items about one receiver. Data were analyzed using a one-way ANOVA test in SPSS 17.0, and means were compared using L-S-D test at the 1 or $5 \%$ confidence level. Figures were drawn by Sigma plot 10.0.

\section{RESULTS AND DISCUSS}

The magnitude of seed germination and germination index are the prerequisite whether a new species could settle down and establish species group successfully. Fig 1 (a) and (c) showed that both the stalk extracts and leaf extracts revealed different extend allelopathy to Brassica campestris, Raphanus sativus L., Oryza sativa, and Lactuca sativa. The two extracts show inhibition effect on Brassica campestris, Raphanus sativus L., and Oryza sativa, and the inhibitory ability was in direct proportion to the concentration of the extracts; to the Lactuca sativa seeds was inhibited at higher concentration and enhanced at lower concentration. Fig 1 (b) and (d) the higher concentration showed the significantly inhibition to the Brassica campestris $(\mathrm{P}<0.05)$, and the strength of leaf extracts was stronger than the stalk extracts. From the Fig $1(\mathrm{a} \sim \mathrm{d})$, the two extracts existed difference among the four receiver seeds. At the same concentration condition, the sequence of seed germination: Brassica campestris $>$ Raphanus sativus L. > Lactuca sativa $>$ Oryza sativa, and leaf extracts $>$ stalk extracts .
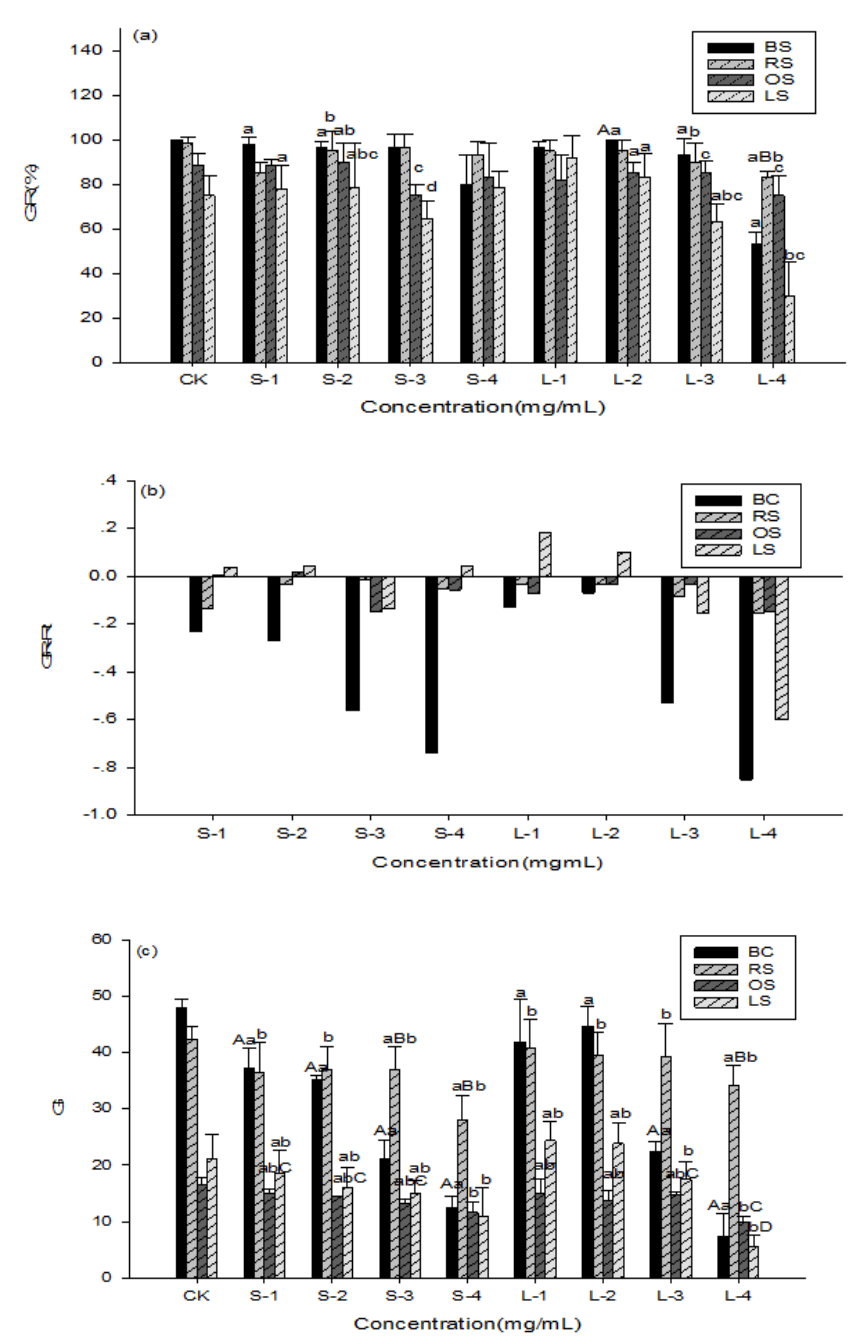


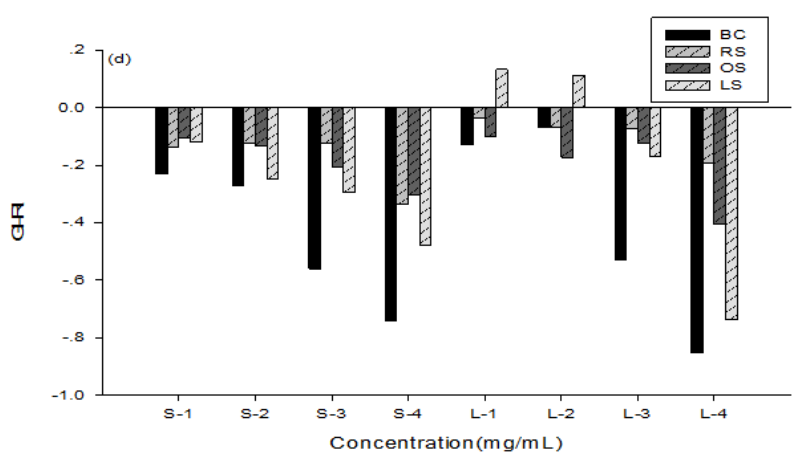

Figure 1. Effect of $H$. rhomboidea extracts on seed germination

Note : a ) Brassica campestris-BC, Raphanus sativus L.-RS, Oryza sativa-OS, and Lactuca sativa -LS; control group -CK; b) values with different lowercase letters mean the same haddle but different concentration difference significant difference, values with different capital letters mean the same handle but different concentration significant difference ; c) S-1 4 and L-1 4 represent the concentration of stalk and leaf were $0.0125,0.025,0.05,0.1 \mathrm{~g} / \mathrm{mL}$ respectively . The same below.

The Fig.2. (a d) showed that the sequence of the two extracts acted on the root length of the four plants was $\mathrm{BC}>\mathrm{RS}>\mathrm{LS}>\mathrm{OS}$; and leaf extracts $>$ stalk extracts, and inhibited at higher concentration and enhanced at lower concentration. Especially the inhibition effect has obvious to the root length of $\mathrm{BC}$ $(\mathrm{P}<0.05)$. From the Fig. 2 (c) $(\mathrm{d})$, the result has been showed that both two extracts have obvious inhibition effect between $\mathrm{BC}$ and $\mathrm{CK}(\mathrm{P}<0.05)$.

The Fig. 3 (a d) showed that both the two extracts revealed different extend allelopathy to the four plants, and the inhibitory ability was in direct proportion to the concentration of the extracts. Inhibition on OS was significant $(\mathrm{P}<0.05)$, on $\mathrm{BC}$ was extremely significant $(\mathrm{P}<0.01)$. The Fig. $3(\mathrm{e} \sim \mathrm{h})$ showed that the inhibition of the two extracts on the fresh weight of RS was significant $(\mathrm{P}<0.05)$, and in direct proportion to the concentration, enhanced to $\mathrm{BC}$ and $\mathrm{OS}$, but not significant $(\mathrm{P}>0.05)$. The allelopathy of two extracts were inhibited at higher concentration and enhanced at lower concentration to the dry weight of $\mathrm{BC}$ and $\mathrm{OS}$, and it was significant $(\mathrm{P}<0.05)$; the extracts of stalk has shown promotion effect on the dry weight of RS, it was significant $(\mathrm{P}<0.05)$, and it was positive with concentration; the extracts of leaf were inhibited at higher concentration and enhanced at lower concentration to the dry weight of RS.

Comprehensive evaluated the different the aqueous extracts of allelopathy with SE, which is the mean value of RI germination, germination index, root length, shoot length, fresh weight and dry weight. The results were shown in Fig. 4, the two extracts showed the different extend inhibition effect on the four receiver, almost characterized lower to enhanced and higher inhibited. The extracts of stalk showed the strongest inhibition on RS, and the leaf showed the strongest inhibition on BC. From different parts extracts, the inhibition of leaf extracts was stronger than the stalks.
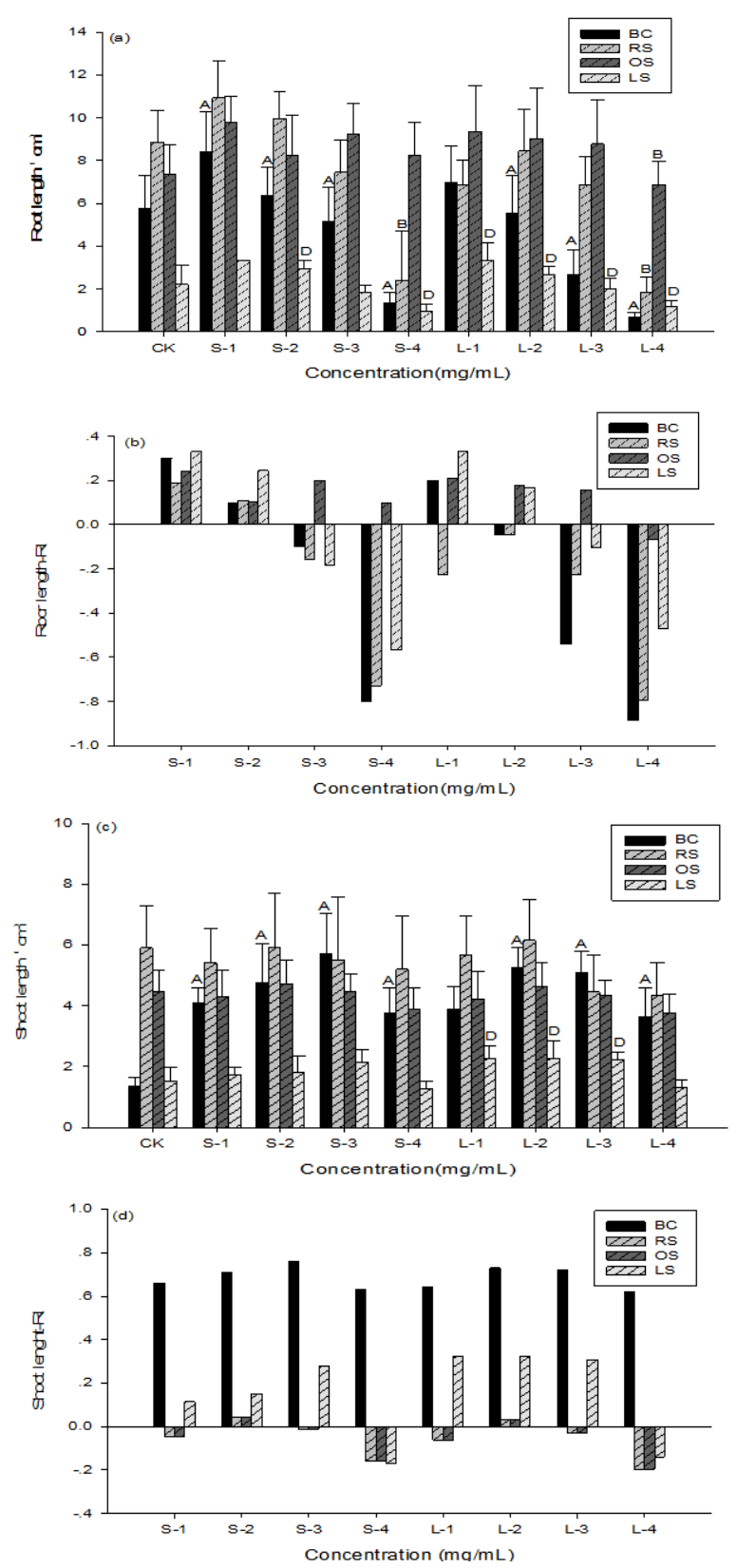

Figure 2 Effects of $H$. rhomboidea extracts on the root length and shoot length
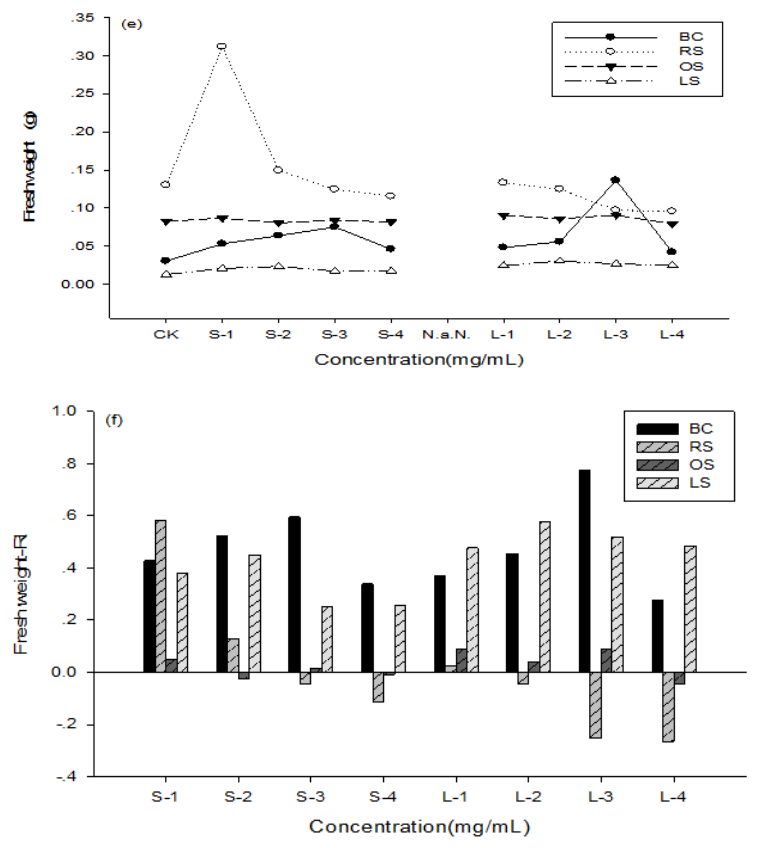

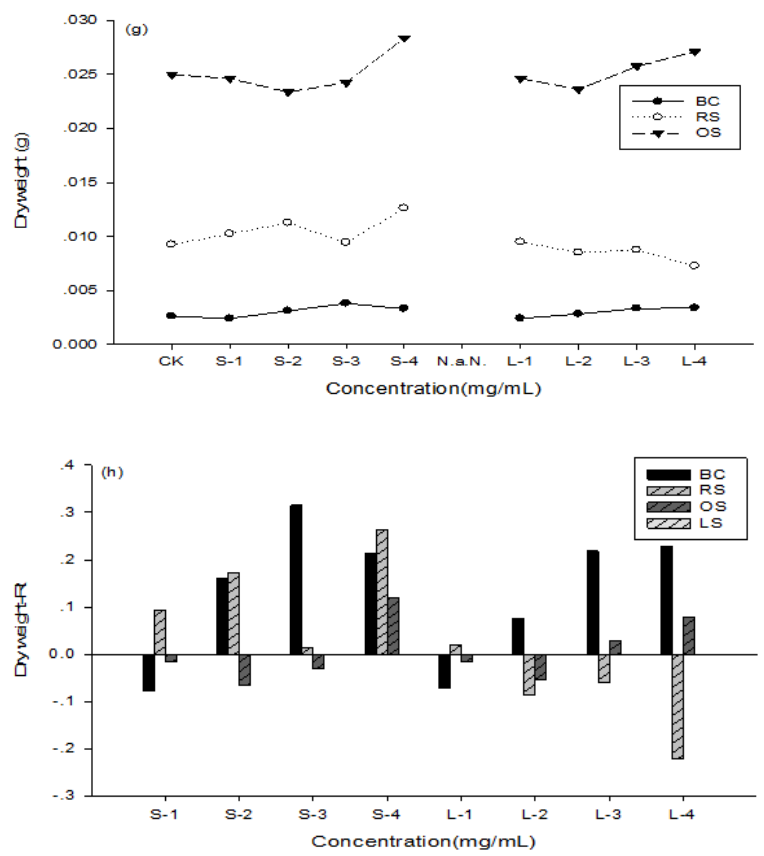

Figure 3 Effects of $H$. rhomboidea extracts on seedling growth

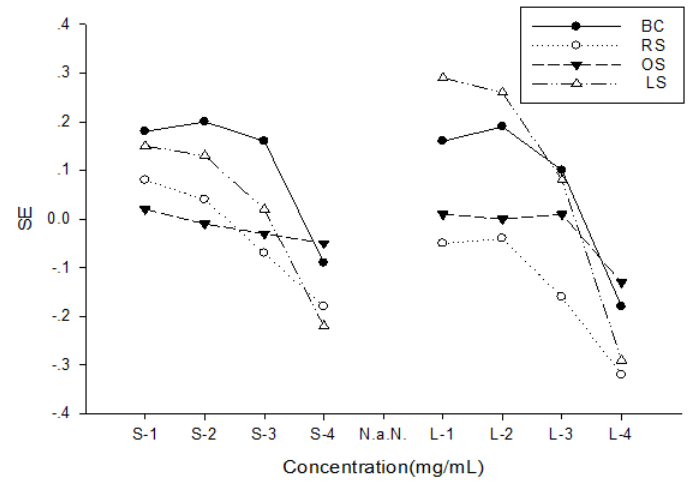

Figure $4 \mathrm{SE}$ of water extracts from $H$. rhomboidea in four receiver plants

\section{CONCLUSION}

The results of this trial has showed that the aerial parts of $H$. rhomboidea existed allelopathy effect. Many studies have shown that the different parts of same plant has the different allelopathy effect, which might be relevant with the different kinds and contents allelochemicals[10-11]. According to the literature reports [12-14]: The common allelochemicals were as fellows: Water soluble organic acid, aliphatic alcohols, aliphatic series, aldehyde, ketone, simple unsaturated lactone, long chain fatty acid, acetylene, quinone compounds, simple phenols, benzoic acid and its derivatives, cinnamic acid and its derivatives, coumarin, flavonoids, tannins, terpenoids, steroids, amino acids, peptides, alkaloids, cyanohydrin together, sulfide, sulfur, nucleotides. The phenolic acid and flavonoids is a common type. Same concentration condition, the inhibition effect of leaf extracts was stronger than stalk in the four receiver plants, that is, the leaves might be more abundant of allelochemicals.

The results indicated that the two extracts from the aerial parts of $H$. rhomboidea had inhibitive effect on the seed germination, and the allelopathy strength increased with the concentration obviously. The inhibition effect of dicotyledons was stronger than monocotyledons, inhibition on Cruciferous was stronger than Compositae, in the same family, the inhibition on $\mathrm{RS}>\mathrm{BC}$. That's indicated that the sensibility to existence in different plants and family of $H$. rhomboidea was different

After the allelochemicals were released in environment, they would be decomposed or degraded, which affected the set free of allelochemicals[15]. $H$. rhomboidea belongs to aromatic plants in Labiatae family, that might be containing volatile substances such as sesquiterpenoids composition[16], which could be set free and act on native plants, but there would be more further study on the mechanism. This trial was under the laboratory conditions, which has a bigger difference with the natural environment, such as natural environment conditions, physical-chemical property of soil,etc. Therefore, because of complexity of allelopathy, not which allelochemicals take effect, and which is the mechanism, but also should combine lab and field, and this lab will continue to further comphensive study on $H$. rhomboidea.

\section{REFERENCES}

[1] Callaway RM, Aschehoug ET, Science, 290:521, 2000.

[2] Bais HP, Vepachedu R, Gilroy S, et al. Science, 301: 1377, 2003.

[3] Lovett JV, Ryuntyu MY, Liu DL, Chem Ecol, 15:1193, 1989.

[4] INDERJIT, CALLAWAYRM. Plant Soil, 256:1, 2003.

[5] Yan F, Yang ZM, Acta Ecologica Sinica, 20:692, 2000.

[6] Salih Mutlu E•Ö kkes Atic. Acta Physiol Plant, 31, 89 (2009)

[7] Tefera T. J Agron Crop Sci, 188:306, 2002.

[8] LEATHER GR, EINHELLIG FA. The Science of Allelopathy. New York:John Wiley \&Sons, 133: 32, 1986.

[9] Williamson GB, Rchardson D, Journal of Chemical Ecology, 14:181, 1988.

[10] Zhang D, Zhang J, Yang W, Wu F. Ecol Res, 25:13, 2010.

[11] Lrababah MA, Tadros MJ, Samarah NH, Ghosheh H. New Forest, 38:261, 2009.

[12] Song J, Chinese Journal of Ecology. 9:43, 1990.

[13] Sun WH, Yu SW. A Plant Physiology Communications. 28:81, 1992.

[14] Wang C X. Procedia Engineering, 18:240, 2011.

[15] Fageria NK, Baligar VC, Upland rice and allelopathy, 34:1323, 2003.

[16] Xu DH, Huang Y S, Jiang DQ, Yuan K, Pharmaceutical Biology, 5:1125, 2013. 